\title{
Jonah Lehrer (2012) Imagine: How CReativity Works
}

\author{
Nelson Zagalo \\ Universidade do Minho, Portugal; nzagalo@ics.uminho.pt
}

Imagine: How Creativity Works (2012) é o terceiro livro de Jonah Lehrer. Depois de Proust was a Neuroscientist (2007) e How We Decide (2009), dois livros de divulgação na área das neurociências, traz-nos agora um trabalho à volta das questões da criatividade. Um livro que faz todo o sentido no seguimento de How We Decide, porque a criatividade no fundo, não é mais do que um processo refinado de tomada de decisões, de resolução de problemas.

Em termos gerais o livro é muito interessante, condensa muito daquilo que se tem estudado sobre o assunto em poucas páginas, carregadas de exemplos e estudos que suportam o que se vai alegando. Apesar de ser um bom livro, fica algo abaixo daquilo a que Lehrer nos habituou. Sente-se o estilo da escrita a aproximar-se demais de alguns bestsellers de divulgação de ciência, como os livros de Malcolm Gladwell ou Daniel Pink, que pegam em meia dúzia de casos e constroem um livro a partir desses casos. Nos seus livros anteriores, Lehrer usava os casos apenas como mote para ir ao fundo das questões, para sobre eles trabalhar a forma como ele próprio vê a realidade. Dando a sua visão pessoal, contribuindo para um avanço do pensamento. Aqui por vezes parece que se fica por agregar casos que suportem uma ideia, sem depois a aprofundar na sua visão pessoal.

Ainda assim Imagine não é um livro de receitas, mas em certa medida pode quase funcionar como tal. Em termos específicos existem vários momentos altos no livro, que diga-se, lê-se muito rapidamente e fluidamente. Passo assim em revista aqui alguns dos pontos que mais me interessaram desta leitura.

\section{Analisar de fora (OUtSider)}

O livro começa com o caso de R\&D do produto Swiffer. Depois dos PhDs em química terem chegado à conclusão que não era possível melhorar mais os produtos de limpeza, a Procter \& Gamble resolveu fazer outsourcing numa empresa de design. Pedindo especificamente um produto novo. Estes, ao contrário dos químicos, não foram ver como melhorar o produto, mas foram antes tentar perceber como é que a atividade era realizada, passaram 3 anos a estudar os comportamentos das pessoas que limpam o chão. Gravaram e viram centenas de horas de vídeo, até que um dia viram alguém usar papel de cozinha meio-húmido para limpar o sujo e deitar fora. E foi aqui que se deu a epifania para criar o Swiffer.

O que a Procter \& Gamble aprendeu com tudo isto foi que as soluções por vezes têm de vir de pessoas não especialistas, pessoas de fora do meio. Neste sentido Lehrer dá também o exemplo da $3 \mathrm{M}$ uma das empresas que mais patentes tem criado nos últimos anos, porque praticamente se dedica apenas à inovação e ao desenvolvimento de novas ideias para outras empresas. E o que estes fazem no seio da $3 \mathrm{M}$ é bastante peculiar. Possuem pessoas de áreas científicas muito distintas que rodam regularmente entre distintas áreas, 
mesmo que nada tenham a ver com elas. Para além disso existem processos na empresa que sugerem o transporte de técnicas de umas áreas para outras. Sem dúvida que a 3M merece um estudo em profundidade relacionado com as noções de transdisciplinaridade.

Com tudo isto a Procter\&Gamble e outras empressa resolveram criar o site Innocentive. Neste site depositam os problemas que as suas equipas de I\&D não conseguem resolver. E esperam que apareça alguém que seja capaz de oferecer uma solução. Pode parecer uma forma de outsourcing barata, uma vez que no fundo não há investimento. Mas não é disso que se trata. O que está aqui em questão é garantir que pessoas que nada têm que ver com aquelas áreas possam surgir com uma ideia a partir de um ponto nunca antes imaginado possível. É algo que vai muito para além dos focus groups ou inquéritos, porque podemos ter milhares de pessoas a olhar para o problema de ângulos inimagináveis. E só isso per se garantirá à partida avanços e inovação. Aliás Lehrer dá o caso de um físico que resolvia problemas de química,

"Ed Melcarek, a seven-time solver on InnoCentive, perfectly exemplifies this finding. Although Melcarek has a master's degree in particle physics, he has never solved a physics challenge on InnoCentive. Instead, he peruses the chemistry and engineering categories on the site, searching for problems that might benefit from his expertise." (Lehrer, 2012, Loc. 1745)

\section{Epifania e Serendipidade}

A meio do livro Lehrer tenta definir mais em concreto o conceito de criatividade, acabando por o rotular de momento de epifania. Aquele momento em que a nossa mente vê claramente a ideia cristalizada, em que "se faz luz". Um momento que é normalmente precedido de serendipidade na associação de ideias mentais. Lehrer fala nas ondas Alfa, que se verificam nos momentos que precedem a epifania. É como se estas varressem o nosso cérebro à procura de ligações, até descobrir o caminho entre ligações correcto. Quando o encontram dá-se a epifania. Ao que parece estas ondas alfa não se activam de modo igual em todos nós, e parece que as pessoas que produzem doses mais elevadas destas ondas são normalmente mais criativas, mais capazes de gerar novas ideias.

Em termos menos técnicos, Lehrer define de forma muito interessante o que diferencia a epifania do pensamento analítico, dedutivo ou indutivo. A ideia aparece-nos à mente de modo quasi-instântaneo, como que empurrada pela serendipidade, enquanto no analítico sentimos claramente a nossa mente a deambular por entre ideias e pensamentos em busca de soluções lógicas.

\section{Trabalho e Foco}

Mas a criatividade não é, de todo, apenas fruto da serendipidade, e de ondas alfa. Lehrer dá-nos muitos exemplos ao longo do livro que demonstram o quanto a criatividade advém e muito do trabalho duro e persistente. 


\begin{abstract}
"The reality of the creative process is that it often requires persistence, the ability to stare at a problem until it makes sense. It's forcing oneself to pay attention, to write all night and then fix those words in the morning. It's sticking with a poem until it's perfect; refusing to quit on a math question; working until the cut of a dress is just right. The answer won't arrive suddenly, in a flash of insight. Instead, it will be revealed slowly, like a coastline emerging from the clouds." (Lehrer, 2012, Loc. 875)
\end{abstract}

Exemplos disto são a quantidade enorme de cientistas, artistas e outros que tomavam drogas para acelerar o seu trabalho, para se manterem acordados, tudo em nome da persistência da busca pelas respostas. E daqui Lehrer vai falar-nos de um caso muito interessante que é o de Clay Marzo, um surfista campeão mundial com Síndrome de Asperger. O que o Asperger faz é normalmente levar a pessoa a evitar o contacto social e a concentrar-se intensamente numa actividade. Neste caso Marzo só consegue estar bem consigo próprio estando dentro de água, e a surfar. Pode passar mais de 8 horas diárias a fazê-lo.

E é isto que faz a diferença, alguém que treina, e treina, e treina vai tornar-se cada vez melhor. Não porque é um criativo, com excesso de ondas Alfa, mas por focar-se, treinar, experimentar, testar, tão intensivamente que acabará por conseguir desenvolver qualidades que os outros não conseguem. E a verdade é que se procurarmos por doentes com Asperger vamos encontrar muitos que se deram muito bem na vida. Apesar de não estarem identificados como tal, muitos acreditam por exemplo que Bill Gates, Steven Spielberg, Mark Zuckerberg entre outros sofrem de Asperger. A razão é a sua declarada inabilidade para lidar com o social, e a sua obsessão com aquilo que fazem. Estes possuem um problema que os leva a focar todas as suas energias apenas naquilo que lhes interessa, e podem por isso conseguir destacar-se.

Isto não quer dizer que o Asperger seja uma bênção. Um doente com asperger pode focar-se em coisas que não são de todo relevantes para a nossa sociedade. Por exemplo saber os nomes todos de listas telefónicas, ou contar folhas de árvores. Ou seja, o que nos diz este ponto, é apenas e só, que a capacidade de nos focar-nos intensamente sobre algo pode ajudar em muito ao desenvolvimento de ações criativas, originais, que inovam.

\title{
4. Deixar Fluir
}

Neste ponto Lehrer trabalha sobre as questões da limitação do nosso córtex préfrontal, que já tinha discutido em How we Decide, e dando vários exemplos que se poderão ler no livro. O que nos interessa aqui reter é o facto de o nosso córtex pré-frontal ser limitado em termos de quantidade de informação que consegue processar. Ou seja enquanto estamos totalmente conscientes não conseguimos lidar com mais do que cinco a sete elementos simultaneamente. Existem drogas que ajudam, os chamados desinibidores, e existem pessoas que conseguem de algum modo suspender esse controlo do córtex pré-frontal, embora isto tenha as suas consequências no resto dos comportamentos. 
"The lesson of letting go is that we constrain our own creativity. We are so worried about playing the wrong note or saying the wrong thing that we end up with nothing at all, the silence of the scared imagination. While the best performers learn how to selectively repress their inhibitions, to quiet the DLPFC [Dorsolateral Prefrontal Cortex] on command, it's also possible to lose one's inhibitions entirely. The result is always tragic, but it's a tragedy often limned with art." (Lehrer, 2012, Loc. 1519)

O nosso sistema DLPFC é dos últimos a desenvolver-se integralmente durante a nossa infância. Por isso existem ideias como a de Picasso "Every child is an artist. The problem is how to remain an artist once we grow up". Ou seja em criança os nossos sistemas de censura não estão activos, e à medida que vamos crescendo vamo-nos tornando cada vez mais conscientes impossibilitando o improviso, ficando demasiado preocupados com o dizer a coisa errada no momento errado. Como refere Lehrer "It's at this point that the infamous "fourth-grade slump" in creativity sets in, as students suddenly stop wanting to make art in the classroom."

Ainda assim podemos sonhar com a fluição de ideias mesmo em adultos, temos é de saber como. Por exemplo o passear livremente pela cidade experienciando as suas sensações, aquilo que Baudelaire qualificou de actividade de Flanêur, podem ser momentos que ajudam a exponenciar a criatividade. Porque é nesses momentos de despreendimento, de deixar fluir, que somos capazes de estabelecer mais pensamentos associativos entre ideias que jazem no nosso inconsciente. Aliás alguns estudos feitos sobre a sesta, demonstram o quão positiva esta é em termos criativos, por permitir esses momentos de relaxe e abertura ao inconsciente na troca de ideias.

"Once we fall asleep, the prefrontal cortex shuts itself down; the censor goes eerily quiet. Meanwhile, neurons all across the brain start shooting out squirts of acetylcholine. But this isn't the usual excitement of reality; this activity is semi-random and unpredictable. It's as if the mind is entertaining itself with improv, filling nighttime narratives with whatever spare details happen to be lying around." (Lehrer, 2012, Loc. 1533)

\section{Social e Small Talk (Pixar)}

Num estudo realizado sobre os musicais da Broadway chegou-se a uma conclusão que nos parece perfeito senso comum, mas que devemos recordar constantemente.

"creative collaborations have a sweet spot: "The best Broadway teams, by far, were those with a mix of relationships," Uzzi says. "These teams had some old friends, but they also had newbies. This mixture meant that the artists could interact efficiently — they had a familiar structure to fall back on — but they also managed to incorporate some new ideas. They were comfortable with each other, but they weren't too comfortable"'. (Lehrer, 2012, Loc. 1988) 
Ou seja para que possamos ser mais criativos, não devemos estar apenas rodeados de grandes amigos, nem de grandes desconhecidos, precisamos de uma mistura saudável. Por outro lado não basta juntar as pessoas de qualquer forma e esperar que estas colaborem apenas e só. Para isso Lehrer dá o excelente exemplo da Pixar, e dos seus métodos de trabalho. A Pixar lançou-se na construção de um novo edifício que foi totalmente pensado por Steve Jobs para poder estimular a criatividade dos criadores da Pixar. Nesse sentido em vez de criarem 3 edifícios separados, foi criado apenas um, e foi criado um enorme hall no centro do edifício de modo a permitir que todos se encontrassem. Para Jobs a questão central de uma empresa passava pela estimulação de interação entre os seus empregados.

But Jobs realized that it wasn't enough simply to create an airy atrium; he needed to force people to go there. Jobs began with the mailboxes, which he shifted to the lobby. Then he moved the meeting rooms to the center of the building, followed by the cafeteria and coffee bar and gift shop. But that still wasn't enough, which is why Jobs eventually decided to locate the only set of bathrooms in the atrium. "'. (Lehrer, 2012, Loc. 2081)

Jobs acreditava que os melhores encontros acontecem por acidente, no hall, no estacionamento, no bar. Jobs sabia que a chamada small talk não era uma perda de tempo, que as conversas aleatórias seriam uma fonte constante de novas ideias. E este tipo de ambiente é o que podemos hoje encontrar em empresas como a Apple, a Google ou a 3M. Como disse Brad Bird o criador de Incredibles e Ratatouille

"The atrium initially might seem like a waste of space ... But Steve realized that when people run into each other, when they make eye contact, things happen. So he made it impossible for you not to run into the rest of the company." (Lehrer, 2012, Loc. 2088)

\section{Brainstorm e a CRítica É fUndamental na CRiatividade}

Um outro ponto importante no livro de Lehrer e que é ainda trabalhado na questão dos métodos de trabalho da Pixar, tem que ver com um dos métodos mais utilizados no mundo das técnicas de criatividade, na técnica de brainstorm. Vários estudos têm mostrado que esta técnica não é particularmente feliz quando comparada com outras, ou mesmo quando comparada com indivíduos a trabalhar isoladamente. Ainda assim, acreditamos particularmente no seu potencial, mas seguindo a lógica que segue a Pixar, que é uma lógica muito herdada da personalidade de Steve Jobs.

Ou seja, o que Alex Osborn nos disse sobre a sua ideia do brainstorm é que esta devia ser aplicada de forma a evitar a crítica. Ou seja juntar as pessoas e levá-las a regurgitar tudo o que lhes vai na mente, sem o receio de que alguém as criticasse. Isto faz algum sentido quando pensamos no ponto acima discutido sobre o "Deixar Fluir". O problema é que os estudos têm demonstrado que as ideias que surgem dos normais processo de brainstorm são em número e qualidade reduzidas. 
Na Pixar, todos os dias de manhã existem reuniões de brainstorm, mas com uma nuance muito distinta de Osborn, é que aqui todos devem contribuir, criticando aquilo que está mal feito. Apontando os defeitos, chamando as coisas pelos nomes. O problema de um brainstorm deste tipo é que tem de ser muito bem gerido e regrado, porque corre o risco de rapidamente resvalar para a agressividade. Nesse sentido a Pixar impõe a seguinte conduta, denominada de Plussing. Uma ideia muito simples, que passa por, sempre que alguém critica alguma coisa, essa crítica deve conter um Plus, ou seja uma nova ideia que ajude a combater o problema encontrado. Segundo Charlan Nemeth, psicólgoca na UC-Berkeley, o que acontece é que,

"the reason criticism leads to more new ideas is that it encourages us to fully engage with the work of others. We think about their concepts because we want to improve them; it's the imperfection that leads us to really listen." (Lehrer, 2012, Loc. 2233)

Aliás é por causa disto que as provas de doutoramento ou mestrado, ou os processos de revisão de artigos dos nossos pares, são tão importantes, nomeadamente quando trabalhados numa perspectiva crítica. É que para podermos apontar uma crítica a um trabalho temos de nos envolver totalmente com ele, temos de entrar dentro da cabeça do seu autor, e pensar como ele, ver a raiz do problema e procurar a sua solução. Se for apenas para dizer bem, esse processo nunca chega a acontecer. Envolver-nos com o trabalho significa que não só o aluno vai ganhar, mas também o professor ganha.

Charlan Nemeth realizou mais alguns estudos que demonstram a raiz do problema do brainstorming tradicional, e que passa pelo facto de o nosso cérebro não funcionar muito bem em termos de livre associação de ideias. Temos tendência para associar ao comum, fácil e familiar. Se perguntam por cor azul, o nosso cérebro diz Céu, ou Oceano. O interessante é a sugestão descoberta por Nemeth para evitar estes buracos do nosso pensamento associativo, e que passa por estimular as pessoas com ideias contrárias, mesmo que erradas, mas que nos façam tirar do marasmo do cliché.

Num dos seus estudos, colocou sujeitos a dizer as cores dos slides que passavam na tela, e no meio dos sujeitos colocou um colaborador, que de vez em quando gritava umas cores ao lado, ou menos usuais, como em vez de vermelho, dizer rosa, ou em vez de azul, dizer turquesa. Quando a seguir questionou as pessoas que tinham sido expostas à sessão com o colaborador que emitia respostas contrárias ou à margem, as pessoas reagiam com respostas mais invulgares que o grupo que não tinha sido exposto ao colaborador. Ou seja à pergunta associativa para azul, já não diziam céu ou oceano, mas diziam por exemplo Smurfs ou tarte de amora.

\section{A FRICÇÃo HUMANA E NÃO A CIDADE}

Este último ponto que aqui discuto é aquele com que mais discordamos do pensamento de Lehrer. Este monta todo um discurso para justificar o facto de que as Cidades são por natureza mais criativas que os meios pequenos, as aldeias. Lehrer suporta-se em Geoffrey West que nos diz que, 
"As cities get bigger, everything starts accelerating. Each individual unit becomes more productive and more innovative. There is no equivalent for this in nature. Cities are a total biological anomaly. But you can't understand modern life without understanding cities. They are the force behind everything interesting. They are where everything new is coming from." (Lehrer, 2012, Loc. 2589)

É verdade que concordamos com a necessidade de "fricção urbana", esta pode ser muito útil e benéfica. Aliás responde àquilo que Jobs professava, de todos se encontrarem com todos, da small talk, do inesperado. Mas não podemos tão facilmente extrapolar isto de um grupo de pessoas para uma grande cidade. E o maior problema é que isso está à vista, se por exemplo Silicon Valley é um dos maiores centros criativos do mundo, por outro lado cidades gigantescas na China, México ou Brasil não se tornam automaticamente criativas apenas através da sua densidade populacional. Lehrer admite que estas discrepâncias entre cidades existem e procura trabalhar o problema realizando uma interessantíssima comparação, mas na qual falha para mim o seu objectivo. Realiza uma comparação entre a "Route 128" em Boston e Silicon Valley, segundo Vivek Wadhwa, professor da Duke

"If you were betting on an area to dominate [the tech sector] in 1975, you'd have been wise to bet on Route 128. It had a giant head start over everywhere else. The region had several elite research universities, such as MIT and Harvard, and a long list of successful technology firms. These companies had big contracts with the Defense Department and controlled the market for microchips and electronic hardware." (Lehrer, 2012, Loc. 2660)

A verdade é que a história não deu razão a este pensamento. Aliás este caso de Leher está mal desde o ponto de partida, porque Moutain View em San Jose, era um lugar agrícola em 1956 quando William Shockley o co-criador do transístor aí se instalou para abrir a Shockley Transistor Corporation, que viria a impulsionar a criação da Intel. Vejam o que é hoje Mountain View, o que demonstra que foi possível gerar grande criatividade num meio pequeno. Para além disso, Mountain View tinha nessa altura uma população de 6 mil pessoas, e em 2010 apesar de todo o desenvolvimento tecnológico, e patentes criadas, está abaixo das 100 mil pessoas, longe, muito longe de ser uma grande cidade.

Depois Lehrer vai tentar demonstrar que o problema da Route 128 ter perdido para Silicon Valley se deveu ao facto de esta ser dominada por empresas gigantes que preservavam segredo de tudo o que faziam e que com isso impediam a criatividade de brotar. Ao contrário de Silicon Valley aonde as pequenas empresas dependiam umas das outras para se fazerem valer, e que por isso partilhavam muitas ideias. O que é em parte verdade, a partilha criativa é um enorme estímulo à criatividade, mas não é o único caminho.

Para fechar o assunto Lehrer dá o exemplo do aparecimento da Apple baseado no sistema de partilha, que é verdade. Nisso Wozniak era totalmente diferente de Jobs, 
tinham visões muito diferentes. Mas a verdade é que a marca criativa deixada pela Apple, não é o Apple I e Il de Wozniak, mas o Macintosh e o iPhone, que por sinal foram criados em grande segredo por Steve Jobs. A diferença é que esses produtos foram criados em segredo, mas por uma equipa de pessoas que trabalhava sob um ambiente criativo igual ao que foi discutido acima no caso da Pixar.

Jobs era extremamente indelicado e rude nas críticas que fazia aos seus colaboradores, mas a verdade é que exigia destes que também fossem críticos e exigentes para com ele. Nas suas reuniões, não era anormal existirem grandes discussões e perturbação emocional entre as pessoas, e estas insurgirem-se contra as ideias de Jobs. Claro que se o fizessem teriam de ter argumentos para sustentar a sua crítica. E talvez seja esse um dos maiores segredos da história da Apple. A discussão profundamente crítica e exigente dos mais ínfimos detalhes dos produtos em desenvolvimento.

Para fechar, este não é o derradeiro livro sobre Criatividade, é antes uma porta para o mundo de ideias que vem aumentado nos anos recentes. Na sua essência, a criatividade é a originalidade, sempre diferente, não padronizável. Aliás como o próprio Jonah Lehrer admite quase no final do livro "every creative story is different".

\section{NOTAS:}

1. Partes deste texto foram previamente publicadas no blog do autor Virtual Illusion.

2. Apesar dos problemas que decorrem das acusações de plágio sobre esta obra, noticiadas na imprensa (http://virtual-illusion.blogspot.pt/2012/og/jonah-lehrer-forjou-citacoes.html), acreditamos que a obra continua a representar uma mais valia, e que a seu tempo essas acusações poderão ser esclarecidas.

\section{REFERÊNCIAS:}

Lehrer, Jonah, (2007), Proust was a Neuroscientist, Houghton Mifflin Harcourt, EUA

Lehrer, Jonah, (2009), How We Decide, Houghton Mifflin Harcourt, EUA

Lehrer, Jonah, (2012), Imagine: How Creativity Works, Houghton Mifflin Harcourt, EUA, Versão Kindle 